\title{
ColoRea: Colour Rearrangement for Dipole Showers
}

\author{
Johannes Bellm* \\ Theoretical Particle Physics, Department of Astronomy and Theoretical Physics, Lund \\ University, Lund, Sweden \\ E-mail: johannes.bellmathep.lu.se
}

In this talk I presented an algorithm to correct for wrong assignment of colour dipoles in the cascade of dipole showers. It is explained how simple matrix elements can be used to reorder the colour connections for the evolution of the following cascade. I discuss the possibility to modify the splitting kernels to include an improved behaviour. Further a numerical study is presented that indicates the insensitivity of reordering with matrix elements of higher multiplicity.

XXVI International Workshop on Deep-Inelastic Scattering and Related Subjects (DIS2018)

16-20 April 2018

Kobe, Japan

${ }^{*}$ Speaker. 


\section{Introduction and Parton Showers}

In this talk I discussed recent findings of reordering dipole chains with simple matrix elements as presented in [1]. Event generators like Herwig7 [2] are important tools to compare experimental data measured at collider experiments like the LHC to theory predictions. Such computer programs contain various components to describe the physics at the high energies $(\mathscr{O}(1 \mathrm{TeV}))$ but also processes that are described at very low energies $(\mathscr{O}(1 \mathrm{GeV}))$. An important part to link between those energy scales in such events are parton shower algorithms. Here the evolution between the high and the low scales are described by probabilistic branching and particle productions. Various algorithms have been developed to describe such processes. Cascades can be produced, as it is done in the angular ordered shower of Herwig7 [2], by successive one-to-two splitting processes. Here the momenta of the contributing partons are reconstructed at some stage of the production. Branching formalisms based on the dipole picture, as e.g. implemented in $[3,4,5,6,7]$, can describe the branching while restoring energy momentum conservation immediately. These algorithms are describe the emissions by two-to-three particles transitions. Here the emitting parton (emitter) $i$ will produce an emission $j$, and in order to conserve energy-momentum locally the momentum of a so-called spectator parton (spectator) $k$ is rescaled ${ }^{1}$. In either case, one-to-two or two-to-three, the cascades are produced probabilistically in the Sudakov veto algorithm with the help of no-emission probabilities constructed with splitting functions and ordering variables. The main differences between the shower algorithms in the two categories are exactly those choices. In the collinear limit $\mathrm{z}$-dependence ${ }^{2}$ the splitting functions need to collapse to the standard Altarelli Parisi splitting functions [8]. In the soft limit an eikonal structure $\left(s_{i k} /\left(s_{i j} s_{j k}\right)\right)$ needs to emerge from the sum over all contributions if the soft gluon $j$ is emitted. The eikonal structure is in the construction of dipole showers shared between the contribution of the emission of the emitter and another possible emission where the roles of emitter and spectator are exchanged.

\section{Starting Conditions and Large $N_{C}$}

In order to start parton shower the conditions need to be set in the beginning. For dipole shower this these are apart from shower starting scales the starting set of dipoles in the leading colour (large $N_{C}$ ) approximation. In this approximation (anti-)quarks are connected to one other parton and gluons are connected to two other partons. These connections are treated as the dipoles described above. This also tells that there is for example one $q \bar{q}$-pair in the process, then the colour connections will form a chain containing all additional gluons and the ends of the chain will be given by the quark and the anti-quark. This configuration is called a dipole chain. For standard processes (excluding loop induced, e.g. Higgs gluon couplings) will have $n$ dipole chains where $n$ is the number of $q \bar{q}$-pairs. If there is no $q \bar{q}$-pair in the process, then the gluons build a cyclic dipole ring. In order to setup the chains the event generator requires information from the matrix element about the large $N_{C}$ structure. In bases like the colour-flow but also the Trace-Basis the

\footnotetext{
${ }^{1}$ If either the emitter or the spectator is part of the initial state of the process the kinematics are modified such that the direction of the initial state parton is preserved. In this case it is also possible that the momenta of other participants of the process at modified.

${ }^{2}$ Where $z$ is the energy fraction of the emitted particle.
} 

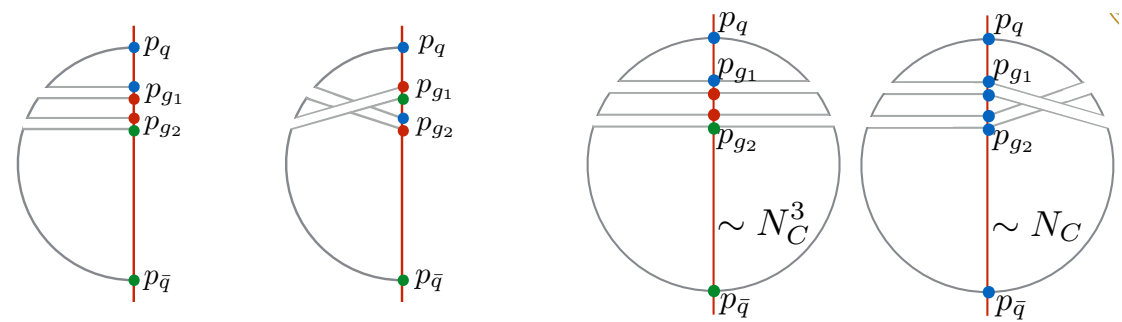

Figure 1: Pictorial representation of possible colour structures. The contraction of different permutations leads to subleading colour configurations.

colour connections can be easily read off. The parton shower starting chains are choosen according to the weight of the squared large $N_{C}$ amplitudes.

\section{Rearranging Chains and Comparison to Data}

While the shower is evolving from hard scales to lower scales the additional emissions need to be inserted in the dipole chains. Here the obvious choice can be the insertion of the emission gluon in between the emitter and the spectator partons. The amplitude in the trace basis with one $q \bar{q}$-pair can be written as (in the notation of [9]),

$$
\mathscr{M}\left(q_{1}, g_{1} g_{2}, \ldots, g_{n}, q_{2}\right)=\sum_{\sigma \in S_{N_{g}}}\left(t^{g_{\sigma_{1}}} t^{g_{\sigma_{2}}} \ldots t^{g_{\sigma_{n}}}\right)_{q_{2}}^{q_{1}} A(\sigma) .
$$

Here $\sigma$ are the permutations of gluons and each permutation can be seen as a possible colour chain configuration. In the amplitude however the kinematics is in sync with the colour part. Note that the kinematic part $|A(\sigma)|$ is in this case used to arrange the colour connections if the shower is started from this stage. Parton shower algorithms are build such that they approximate the next higher multiplicity. So if we insert a "new" gluon into this chain we need to take care that the kinematic part is still in sync with the colour part. A main source of ambiguity of appointing the ordering after the emission of gluon is the splitting of a gloun into a pair of gluons, namely the $g \rightarrow g g$ splitting. The AP splitting function reads,

$$
P(z) \sim \frac{z}{1-z}+\frac{1-z}{z}+z(1-z)
$$

where the first part corresponds to a soft emission and the second to a soft emitter. The third part, essentially proportional to the transverse momentum, is purely collinear. In the formalism proposed by Catani and Seymour [10] the subtraction expression for gluon splitting is written such that it reproduces the AP kernels and since the sum is taken over all configurations it reproduces the soft and collinear behaviour of real emission. If we however want to construct a parton shower we need to take care an very soft emission does not modify the other parts of the cascade strongly. A soft emitter, mainly produced by the second part of Eq.3.2, however will alter the kinematics differently than the colour part of the amplitude.

One can now modify the splitting kernel to anti-symmetrize w.r.t. emitter and emission. Two examples are seem reasonable. One can use the kernel multiplied by $2 z$ and therefore essentially 

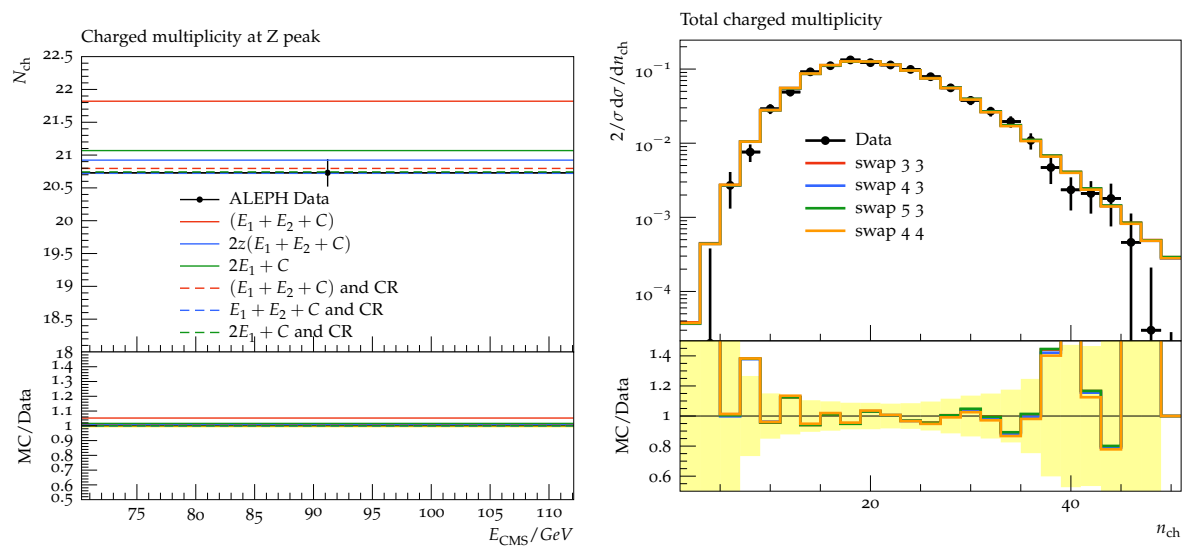

Figure 2: Left plot shows the results for modified $g \rightarrow g g$ splitting kernels, data from [11]. All choices collapse if in addition the ColoRea algorithm is performed. The right plot shows the result if one rearranges the colour dipole chains with matrix elements (ME) of higher multiplicity. Here the first integer gives the ME multiplicity up to which the highest multiplicity is rearranged. The second indicates the ME multiplicity used for higher multiplicity chains. The data is from [12]

tag the harder/emitter gluon. Another possibility is to choose only the first part of eikonal part for the soft emission, multiplied by a factor 2 and keep the purely collinear part unchanged. The third option is to keep the old kernel and then decide the insertion in the dipole chain probabilistically according to $\mathrm{z}$ vs. 1-z. In the left plot in Fig. 2 the choice of symmetrised splitting functions in red and the two options are shown in blue and green. It is clearly visible that the modification has an impact on the result. But the ambiguity remains. In the following an algorithm is proposed in which the chains are rearranged after emissions to resolve the ambiguity by comparing to the leading colour structure of real matrix elements.

In [1] an algorithm was proposed to correct for the disordering of gluon insertions. Here, instead of making the choice between the options above but also to take into account emissions from other legs, simple matrix elements are used to probabilistically rearrange the colour chains in the shower process. To do so the matrix elements for a $\gamma^{*} \rightarrow q \bar{q} g g$ was generated using MadGraph5 [13] and the code was modified to extract the leading colour expressions for the squared amplitudes. Two possible colour structures, depicted in Fig. 1, are possible in this case. The algorithm now probes after each emission probabilistically if the other ordering should be chosen. Here we check if a random number is smaller than the current configuration with weight $\mid\left. A($ cur. $)\right|^{2} /\left(\mid\left. A(\right.$ cur. $)\right|^{2}+$ $\mid\left. A($ other $\left.)\right|^{2}\right)$. If not the ordering is altered. If we have more than the two gluons in the chain the ordering of two gluons can still be tested using the momenta of the then neighbouring gluon(s) in the ME to be the quark momenta. The dashed lines in the same left Fig. 2 as described above are produced using this algorithm.

To test the validity of this approach we can produce matrix elements for more than two gluons and test according to those, see Fig. 3. Then we obviously will have more than the two permutations, but still each permutation can be associated with a weight and the rearrangement can be performed according to this weight. The right plot in Fig. 2 shows the result for the charged particle multiplicity as measured at LEP if one reorders the chains with higher multiplicity matrix elements. 


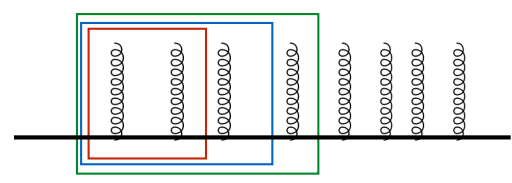

Figure 3: Pictorial representation of correcting dipole chains with matrix elements with two, three or four gluons. The right plot in Fig. 3 shows, in the same colour coding, the result if the same multiplicity is rearranged with the corresponding matrix elements.

The labels indicate if to which order the same matrix element multiplicity was used as the first integer and which matrix elements have been used to correct the ordering if the chain size exceeds the number of available matrix element legs. As can be seen the results are very stable numerically and further investigations on the validity of the methods are ongoing. Other data comparison can be found in [1]

\section{Summary}

In this talk I presented an algorithm that allows to reorder the chains of dipole showers with the leading $N_{C}$ limit of leading order matrix elements. I discussed and showed comparisons of modified spitting kernels and the reordering with matrix elements of higher multiplicity.

Acknowledgements: I thank the organizers of the conference for the hospitality and the nice social program. I acknowledge the funding from the European Research Council (ERC) under the European Union's Horizon 2020 research and innovation programme, grant agreement No 668679.

\section{References}

[1] J. Bellm arXiv:1801.06113.

[2] J. Bellm et al. Eur. Phys. J. C76 (2016), no. 4 196, [arXiv:1512. 01178].

[3] L. Lönnblad Comput. Phys. Commun. 71 (1992) 15-31.

[4] T. Sjöstrand et al. Comput. Phys. Commun. 191 (2015) 159-177, [arXiv: 1410 . 3012].

[5] T. Gleisberg et al. JHEP 02 (2009) 007, [arXiv: 0811. 4622].

[6] S. Plätzer and S. Gieseke JHEP 01 (2011) 024, [arXiv: 0909 . 5593].

[7] S. Höche and S. Prestel Eur. Phys. J. C75 (2015), no. 9 461, [arXiv: 1506.05057 ].

[8] G. Altarelli and G. Parisi Nucl. Phys. B126 (1977) 298-318.

[9] M. Sjödahl Eur. Phys. J. C75 (2015), no. 5 236, [arXiv: 1412 . 3967].

[10] S. Catani and M. H. Seymour Nucl. Phys. B485 (1997) 291-419, [hep-ph / 9605323 ]. [Erratum: Nucl. Phys.B510,503(1998)].

[11] ALEPH Collaboration, A. Heister et al. Eur. Phys. J. C35 (2004) 457-486.

[12] ALEPH Collaboration, D. Decamp et al. Phys. Lett. B273 (1991) 181-192.

[13] J. Alwall, R. Frederix, S. Frixione, V. Hirschi, F. Maltoni, O. Mattelaer, H. S. Shao, T. Stelzer, P. Torrielli, and M. Zaro JHEP 07 (2014) 079, [arXiv: 1405.0301 ]. 